\title{
On a Bulk Queueing System with Impatient Customers
}

\author{
LOTFI TADJ *, LAKDERE BENKHEROUF and LAKHDAR AGGOUN \\ Department of Statistics and Operations Research, College of Science, \\ King Saud University, P.O. Box 2455, Riyadh 11451, Saudi Arabia
}

(Received9 March 1997)

We consider a bulk arrival, bulk service queueing system. Customers are served in batches of $r$ units if the queue length is not less than $r$. Otherwise, the server delays the service until the number of units in the queue reaches or exceeds level $r$. We assume that unserved customers may get impatient and leave the system. An ergodicity condition and steady-state probabilities are derived. Various system characteristics are also computed.

Keywords: Queue; Impatience; Embedded Markov chain; Semi-regenerative process; Steady-state distribution

AMS Subject Classification: Primary: 60K10, 60K25; Secondary: 90B22, 90B25

\section{INTRODUCTION}

We consider a bulk arrival, bulk service queueing system. Customers arrive in batches of random size but are served in batches of a fixed size $r$. The server follows a delayed service discipline in which the processing of customers does not start unless at least $r$ of them are waiting in the queue. We also assume that some (or all) of the unserved customers may get impatient and leave the system.

Queueing systems where the server has to wait for the queue length to accumulate to a fixed level are called service delayed queueing systems. They have been introduced by Abolnikov et al. [2]. Various

\footnotetext{
* Corresponding author. Fax: 011-966-1-467-6274. E-mail: f40t023@ksu.edu.sa.
} 
extensions of these systems were subsequently suggested: for a thorough review see Dshalalow [4]. Transportation systems are examples of such systems since servicing small groups may cause high maintenance costs.

The process of interest is the number of customers in the system at an arbitrary instant of time. We first consider the process embedded over the times of service completions, then we study the general queueing process as a semi-regenerative process. In both cases we establish a necessary and sufficient criterion for ergodicity and find the stationary distribution and various system characteristics. Illustrative examples are provided.

The next section describes the model formally and introduces the notation. Section 3 reviews some theorems and definitions needed in the analysis. Section 4 is concerned with the discrete time parameter queueing process while Section 5 contains the results of the continuous time parameter process.

\section{MODEL DESCRIPTION AND NOTATION}

Assume that there is a single-server queueing system with an unlimited capacity waiting room. All stochastic processes will be defined on the probability space $(\Omega, \mathfrak{F}, P)$.

(a) Input: At time $\tau_{n}$, a batch of size $X_{n}$ arrives to the service facility. We assume that $\left\{X_{n}\right\}$ is a sequence of iid random variables independent of $\tau_{n}$, with probability generating function

$$
a(z)=E\left[z^{X_{1}}\right]
$$

and with finite first moment

$$
a=E\left[X_{1}\right] .
$$

Let $\{N(t), t \geq 0\}$ denote the Poisson process associated with the point process $\left\{\tau_{n}\right\}$ whose jumps occur at a rate $\lambda>0$. Then the input $Z(t)=\sum_{i=1}^{N(t)} X_{i}$ is a compound Poisson process.

(b) Service: Let $\{Q(t), t \geq 0\}$ be a stochastic process describing the number of units in the system at time $t$ and let $\left\{T_{n}\right\}$ be the sequence of 
successive service completions. Also, let $Q_{n}=Q\left(T_{n}+0\right)$. At time $T_{n}$, the server takes a batch of units of size $r$ from the queue and serves it during a random length of time $\sigma_{n+1}$ if the queue length $Q_{n}$ is at least $r$. Otherwise, the server idles until the queue length, for the first time, reaches or exceeds the level $r$. If $Q_{n} \geq r, T_{n+1}-T_{n}$ is equal to $\sigma_{n+1}$. If $Q_{n}<r$, the length of the time interval $\left(T_{n}, T_{n+1}\right]$ is the sum of an idle period and the actual service time $\sigma_{n+1}$. In both cases we assume that $\sigma_{n+1}$ has a probability distribution $B$ with finite mean $b$.

(c) Impatience: When the number $Q_{n}$ of units waiting in line at a service completion is larger than $r$, only $r$ of them will be taken by the server. Then some or all of the remaining $\left(Q_{n}-r\right)$ customers may leave the system. We denote by $\Delta_{n}\left(Q_{n}-r\right)$ the number of those that stay in the queue. Assuming a "stable impatience" of arriving customers once the queue length exceeds some (perhaps large) number $N$, introduce the following conditional probability generating function

$$
g_{i}(z)= \begin{cases}1, & i<r, \\ \sum_{s=0}^{i-r} g_{s}^{(i-r)} z^{s}, & r \leq i \leq N, \\ z^{i-r}, & i>N,\end{cases}
$$

where $g_{s}^{(i-r)}=P^{i}\{s$ customers out of $(i-r)$ stay in the system and wait for the next service $\}, 0 \leq s \leq i-r, i \geq r$. The first moment

$$
\bar{g}_{i-r}=E\left[\Delta_{0}\left(Q_{0}-r\right)\right]
$$

is assumed to be finite.

We recall that the Laplace-Stieltjes transform (LST) $B^{*}$ of a probability distribution function $B$, is defined by

$$
B^{*}(u)=\int_{0}^{\infty} \mathrm{e}^{-u x} B(\mathrm{~d} x), \quad \operatorname{Re}(u) \geq 0,
$$

where $\operatorname{Re}(u)$ stands for the real part of $u$.

\section{PRELIMINARIES}

\subsection{Fluctuation Theory}

Because customers arrive in batches, the queueing process is more likely to exceed rather than hit exactly level $r$. This special feature 
requires a knowledge of the critical behavior of a delayed renewal process about a certain level, which is different from the analysis of orderly processes. This problem, known as the first passage problem, was treated by Abolnikov and Dshalalow [1]. We give below some results from [1] that are needed in the analysis.

Consider the delayed renewal process $S_{n}=\sum_{i=1}^{n} X_{i},\left(S_{0}=X_{0}\right)$ and assume that the processes $\left\{\tau_{n}\right\}$ and $\left\{S_{n}\right\}$ are independent. For a fixed integer $r \geq 1$, we will be interested in the bahavior of $\left\{S_{n}\right\}$ and other related processes.

Definition 1 (Abolnikov and Dshalalow [1]) For each $n$, the random variable:

(i) $\nu_{n}=\inf \left\{k: S_{k} \geq r\right\}$ is called the index of the first excess (above level $r-1)$.

(ii) $S_{\nu_{n}}$ is called the level of the first excess (above $r-1$ ).

(iii) $\tau_{\nu_{n}}$ is known as the first passage time of $S$ above level $r$.

(iv) $\eta_{n}=S_{\nu_{n}}-r$ is called the total increment.

Let

$$
\gamma^{(i)}(z)=E^{i}\left[z^{\nu_{0}}\right], \quad G^{(i)}(z)=E^{i}\left[z^{S_{\nu_{0}}}\right]
$$

Also let

$$
\bar{\nu}^{(i)}=E^{i}\left[\nu_{0}\right], \quad \bar{\tau}^{(i)}=E^{i}\left[\tau_{\nu_{0}}\right], \quad \bar{S}^{(i)}=E^{i}\left[S_{\nu_{0}}\right], \quad \bar{\eta}^{(i)}=E^{i}\left[\eta_{n}\right],
$$

and define the operator

$$
\mathcal{D}_{x}^{k}=\lim _{x \rightarrow 0} \frac{1}{k !} \frac{\partial^{k}}{\partial x^{k}}, \quad k \geq 0 .
$$

Proposition 1 (Abolnikov and Dshalalow [1])

(i) The probability generating function $\gamma^{(i)}(z)$ of the index of the first excess level satisfies the following formula:

$$
\gamma^{(i)}(z)= \begin{cases}z \mathcal{D}_{x}^{r-i-1}\left\{\frac{1-a(x)}{(1-x)[1-z a(x)]}\right\}, & i<r, \\ 1, & i \geq r,\end{cases}
$$

where $a(x)$ is given by (2.1) and $\mathcal{D}_{x}^{k}$ is defined in (3.1). 
(ii) The probability generating function $G^{(i)}(z)$ of the first excess level is determined by the following formula:

$$
G^{(i)}(z)= \begin{cases}z^{r} \mathcal{D}_{x}^{r-i-1}\left\{\frac{a(z)-a(x)}{(z-x)[1-a(x)]}\right\}, & i<r \\ z^{i}, & i \geq r\end{cases}
$$

COROllary 1 (Abolnikov and Dshalalow [1]).

(i) The mean value of the index of the first excess:

$$
\bar{\nu}^{(i)}= \begin{cases}\mathcal{D}_{x}^{r-i-1}\left\{\frac{1}{(1-x)[1-a(x)]}\right\}, & i<r, \\ 0, & i \geq r .\end{cases}
$$

(ii) The mean value of the first passage time:

$$
\bar{\tau}^{(i)}=\frac{1}{\lambda} \bar{\nu}^{(i)}
$$

(iii) The mean value of the level of the first excess:

$$
\bar{S}^{(i)}=i+a \bar{\nu}^{(i)}
$$

with a defined by (2.2).

(iv) The mean value of the total increment:

$$
\bar{\eta}^{(i)}=a \bar{\nu}^{(i)} .
$$

Of interest also is the generator of the first excess level defined by

$$
\tilde{G}_{i}(z)=\sum_{j \geq 0}^{r-i-1} E^{i}\left[z^{S_{j}} I_{U_{r-1}}\left(S_{j}\right)\right],
$$

where $U_{p}=\{0,1, \ldots, p\}$ and $I_{U}$ is the indicator function of a set $U$.

It can be shown that $\tilde{G}_{i}(z)$ is a polynomial of $(r-1)$ th degree that can be determined from the following formula:

$$
\tilde{G}_{i}(z)= \begin{cases}z^{i} \mathcal{D}_{x}^{r-i-1}\left\{\frac{1}{(1-x)[1-a(x z)]}\right\}, & i<r, \\ 0, & i \geq r .\end{cases}
$$


It is related to the generating function $G^{(i)}(z)$ (of the first excess level) by

$$
G^{(i)}(z)=z^{i}-[1-a(z)] \tilde{G}_{i}(z) .
$$

\subsection{Delta Matrices}

We will be proving that the embedded process $\left\{Q_{n}\right\}$ is an ergodic Markov chain. In order to do that, we shall be using the concept of delta matrices introduced by Abolnikov and Dukhovny [3] and some related results.

Definition 2 (Abolnikov and Dukhovny [3]) A finite or an infinite stochastic matrix $A=\left(a_{i j} ; i, j \geq 0\right)$ is called a $\Delta_{m, n}$-matrix, $n \geq$ $m \geq 1$, if $a_{i j}=k_{j-i+r}$ for $i>n, j \geq i-m ; a_{i j}=0$ for $i>n, j<i-m$, where $\left\{k_{i}, i \geq 0\right\}$ is a given probability mass function.

Proposition 2 (Abolnikov and Dukhovny [3]) Let $\left\{Q_{n}\right\}$ be an irreducible aperiodic Markov chain with transition probability matrix $A$ in the form of a $\Delta_{m, n}$-matrix and let $A_{i}(z)$ be the generating function of the ith row of $A$ and $K(z)=\sum_{j=0}^{\infty} k_{j} z^{j}$. Then $\left\{Q_{n}\right\}$ is recurrent-positive if and only if

$$
\left.\frac{\mathrm{d}}{\mathrm{d} z} A_{i}(z)\right|_{z=1}<\infty, \quad i=0,1, \ldots, n
$$

and

$$
\left.\frac{\mathrm{d}}{\mathrm{d} z} K(z)\right|_{z=1}<m
$$

Proposition 3 (Abolnikov and Dukhovny [3]) Under condition (3.11) the function $z^{r}-K(z)$ has exactly $r$ roots that belong to the closed unit ball $\vec{B}(0,1)=\{z \in \mathbb{C}:\|z\| \leq 1\}$. Those of the roots lying on the boundary $\partial B(0,1)$ are simple.

\section{EMBEDDED PROCESS}

\subsection{Probability Generating Function}

The queueing process $\left(Q_{n}\right)$ is a Markov chain. Indeed, let $V_{n}=Z\left(\sigma_{n}\right)$, $n \geq 0$, where $Z(\cdot)$ is the input process. Then using the notation of the 
previous section, we have

$$
Q_{n+1}= \begin{cases}S_{\nu_{n}}-r+V_{n+1}, & Q_{n}<r \\ \Delta_{n}\left(Q_{n}-r\right)+V_{n+1}, & Q_{n} \geq r\end{cases}
$$

It follows that

$$
A_{i}(z)= \begin{cases}z^{-r} G^{(i)}(z) F(z), & i<r \\ g_{i}(z) F(z), & i \geq r\end{cases}
$$

where

$$
F(z)=B^{*}(\lambda-\lambda a(z))
$$

and $g_{i}(z)$ is given by (2.3) and $G^{(i)}(z)$ by (3.3).

It can be shown that the transition probability matrix $A$ of the Markov chain $\left\{Q_{n}\right\}$ is a $\Delta_{r, N}$-matrix. The following is the main result of this section.

\section{Proposition 4}

(i) The Markov chain $\left\{Q_{n}\right\}$ is ergodic if and only if

$$
\rho=\lambda a b<r,
$$

where $a$ is given by (2.2) and $b$ is the mean service time.

(ii) Let $\boldsymbol{P}=\left(p_{i}, i \geq 0\right)$ be the invariant probability measure of operator $A$ and let $P(z)$ be the generating function of vector $\boldsymbol{P}$. Then under condition (4.4):

$$
P(z)=\frac{\sum_{i=0}^{N}\left[z^{r} A_{i}(z)-z^{i} F(z)\right] p_{i}}{z^{r}-F(z)} .
$$

(iii) The unknown probabilities $p_{0}, p_{1}, \ldots, p_{N}$ form the unique solution of the following system of equations:

$$
\left.\sum_{i=0}^{N} \frac{\mathrm{d}^{k}}{\mathrm{~d} z^{k}}\left[A_{i}(z)-z^{i}\right] p_{i}\right|_{z=z_{s}}=0, \quad k=0, \ldots, k_{s}-1 ; s=1, \ldots, S+1,
$$

$$
a \sum_{i=0}^{r-1} \bar{\nu}^{(i)} p_{i}+\sum_{i=r}^{N}\left(r-i+\bar{g}_{i-r}\right) p_{i}=r-\rho,
$$


where $z_{s}$ are the roots of $z^{r}-F(z)=0$ in the region $\bar{B}(0,1) \mid\{1\}$ with their multiplicities $k_{s}$ such that $\sum_{s=0}^{S} k_{s}=r-1$ and $z_{S+1}=0$ is of multiplicity $N+1-r$. Here $\bar{\nu}^{(i)}$ is the mean index of the first excess, $\bar{g}_{i-r}$ is given by (2.4), and $\rho$ by (4.4).

Proof

(i) We use Proposition 2. Relation (3.10) is satisfied and relation (3.11) is equivalent to (4.4).

(ii) Formula (4.5) follows from the fact that $P(z)=\sum_{i=0}^{\infty} A_{i}(z) p_{i}$ and relation (4.2).

(iii) System (4.6)-(4.7) is derived as follows. First, rewrite (3.10):

$$
\sum_{i=N+1}^{N} p_{i} z^{i-(N+1)}=\frac{\sum_{i=0}^{N+1}\left[A_{i}(z)-z^{i}\right] p_{i}}{z^{N+1-r}\left[z^{r}-F(z)\right]} .
$$

The left-hand side of the above relation is analytic in $\bar{B}(0,1) \backslash\{1\}$ and therefore so is the right-hand side. Now the denominator of the right-hand side has $r-1$ simple roots in $\bar{B}(0,1) \backslash\{1\}$ (Proposition 3) and therefore these roots are also roots of the numerator. Call $z_{s}, s=1, \ldots, S$, these roots. Let $k_{s}, s=1, \ldots, S$, be their respective multiplicities such that $\sum_{i=0}^{S} k_{s}=r-1$ and $z_{S+1}=0$ be the $(S+1)$ th root, of multiplicity $(N+1-r)$. These considerations yield system (4.6) of $N$ equations with $(N+1)$ unknowns $p_{0}, \ldots, p_{N}$. One more equation is necessary to obtain a system of $(N+1)$ equations with $(N+1)$ unknowns. We derive it from the identity $P(1)=1$. For $z=1$, both the numerator and denominator in (4.5) are zero. Using L'Hôpital's rule and relation (4.2) we get the last equation, (4.7), of the system.

The uniqueness of $\left\{p_{0}, \ldots, p_{N}\right\}$ can be shown using similar arguments to that of Dshalalow and Tadj [6].

\subsection{Examples}

(1) Consider a special case of the queueing system where the service time has an exponential probability distribution function with parameter $1 / b$. Then $F(z)=\{1+b \lambda[1-a(z)]\}^{-1}$. Different distributions may be assumed for the size of arriving batches. 
(i) Assume $a(z)=z$ (i.e., single arrivals). In this case $a=1$,

$$
G^{(i)}(z)=\left\{\begin{array}{ll}
z^{r}, & i<r, \\
z^{i}, & i \geq r,
\end{array} \quad A_{i}(z)= \begin{cases}F(z), & i<r \\
g_{i}(z) F(z), & i \geq r\end{cases}\right.
$$

and formula (4.5) reduces to

$$
P(z)=\frac{F(z)}{z^{r}-F(z)}\left\{\sum_{i=0}^{r-1}\left(z^{r}-z^{i}\right) p_{i}+\sum_{i=r}^{N}\left[z^{r} g_{i}(z)-z^{i}\right] p_{i}\right\} .
$$

Also in this case

$$
D_{x}^{r-i-1}\left\{\frac{1}{(1-x)[1-a(x)]}\right\}=r-i,
$$

so that

$$
\begin{aligned}
\bar{\nu}^{(i)} & = \begin{cases}r-i, & i<r, \\
0, & i \geq r,\end{cases} \\
\bar{\tau}^{(i)} & = \begin{cases}(r-i) / \lambda, & i, \\
0, & i \geq r,\end{cases} \\
\bar{S}^{(i)} & = \begin{cases}r, & i<r, \\
0, & i \geq r,\end{cases} \\
\bar{\eta}^{(i)} & = \begin{cases}r-i, & i<r, \\
0, & i \geq r .\end{cases}
\end{aligned}
$$

(ii) Assume $a(z)=z^{2}$ (i.e., customers arrive by groups of 2). In this case $a=2$,

$$
\begin{aligned}
G^{(i)}(z) & = \begin{cases}\frac{1}{2} z^{r}\left[z\left(1+(-1)^{r-i-1}\right)+\left(1-(-1)^{r-i-1}\right)\right], & i<r, \\
z^{i}, & i \geq r,\end{cases} \\
A_{i}(z) & = \begin{cases}\frac{1}{2}\left[z\left(1+(-1)^{r-i-1}\right)+\left(1-(-1)^{r-i-1}\right)\right] F(z), & i<r, \\
g_{i}(z) F(z), & i \geq r,\end{cases}
\end{aligned}
$$


and formula (4.5) reduces to

$$
\begin{aligned}
P(z)=\frac{F(z)}{z^{r}-F(z)}\{ & z^{r}\left(\sum_{\substack{i=0 \\
r-i=1=2 l}}^{r-1}\left(z-z^{i}\right) p_{i}+\sum_{\substack{i=0 \\
r-i-1=2 l+1}}^{r-1}\left(1-z^{i}\right) p_{i}\right) \\
& \left.+\sum_{i=r}^{N}\left[z^{r} g_{i}(z)-z^{i}\right] p_{i}\right\} .
\end{aligned}
$$

Also in this case

$$
D_{x}^{r-i-1}\left\{\frac{1}{(1-x)[1-a(x)]}\right\}=\frac{1}{2}(r-i)+\frac{1}{4}\left[1+(-1)^{r-i-1}\right],
$$

so that

$$
\begin{aligned}
\bar{\nu}^{(i)} & = \begin{cases}\frac{1}{2}(r-i)+\frac{1}{4}\left[1+(-1)^{r-i-1}\right], & i<r, \\
0, & i \geq r,\end{cases} \\
\bar{\tau}^{(i)} & = \begin{cases}\frac{1}{2} \frac{r-i}{\lambda}+\frac{1}{4} \frac{1+(-1)^{r-i-1}}{\lambda}, & i<r, \\
0, & i \geq r,\end{cases} \\
\bar{S}^{(i)} & = \begin{cases}r+\frac{1}{2}\left[1+(-1)^{r-i-1}\right], & i<r, \\
0, & i \geq r,\end{cases} \\
\bar{\eta}^{(i)} & = \begin{cases}r-i+\frac{1}{2}\left[1+(-1)^{r-i-1}\right], & i<r, \\
0, & i \geq r .\end{cases}
\end{aligned}
$$

(iii) Assume $a(z)=p z /(1-q z)$ (i.e., the group size follows a geometric distribution with parameter $p$ ). In this case $a=1 / p$,

$$
G^{(i)}(z)=\left\{\begin{array}{ll}
\frac{p}{1-q z} z^{r}, & i<r, \\
z^{i}, & i \geq r,
\end{array} \quad A_{i}(z)= \begin{cases}\frac{p}{1-q z} F(z), & i<r, \\
g_{i}(z) F(z), & i \geq r,\end{cases}\right.
$$

and formula (4.5) reduces to

$$
P(z)=\frac{F(z)}{z^{r}-F(z)}\left\{\sum_{i=0}^{r-1}\left(\frac{p z}{1-q z} z^{r}-z^{i}\right) p_{i}+\sum_{i=r}^{N}\left[z^{r} g_{i}(z)-z^{i}\right] p_{i}\right\} .
$$


Also in this case

$$
D_{x}^{r-i-1}\left\{\frac{1}{(1-x)[1-a(x)]}\right\}=p(r-i)+q,
$$

so that

$$
\begin{aligned}
& \bar{\nu}^{(i)}= \begin{cases}p(r-i)+q, & i<r, \\
0, & i \geq r,\end{cases} \\
& \bar{\tau}^{(i)}= \begin{cases}p \frac{r-i}{\lambda}+\frac{q}{\lambda}, & i<r, \\
0, & i \geq r,\end{cases} \\
& \bar{S}^{(i)}= \begin{cases}r+\frac{q}{p}, & i<r, \\
0, & i \geq r,\end{cases} \\
& \bar{\eta}^{(i)}= \begin{cases}r-i+\frac{q}{p}, & i<r, \\
0, & i \geq r .\end{cases}
\end{aligned}
$$

(2) In example 1 above, $P(z)$ is fully determined only after $p_{0}, \ldots, p_{N}$ have been computed by solving system (4.6)-(4.7). As an illustration, let $r=2$ and $N=4$ in example 1 . According to Proposition 4 , the denominator on the right-hand side of (4.8) is $z^{3}\left[z^{2}-F(z)\right]$. It has $z_{1}=0$ as a root of multiplicity $k_{1}=3$. Finding the roots of $z^{2}-F(z)$ reduces to solving

$$
z^{2}-1-z^{2} b \lambda[a(z)-1]=0 .
$$

Since $a(1)=1$, then $a(z)-1$ can be factored into $(z-1) R(z)$. Equation (4.9) becomes

$$
b \lambda z^{2} R(z)-z-1=0 .
$$

In example 1(i) above, $R(z)=1$ and Eq. (4.10) has root $z_{0}=$ $\left(1-\sqrt{1+4 \rho)} /(2 \rho)\right.$. Substituting $z_{1}$ with $k=0$, 1 , and 2 and $z_{0}$ in (4.6) yields 4 equations which along with (4.7) yield a system of 5 equations in 5 unknown probabilities $p_{0}, \ldots, p_{4}$. A similar system is obtained in example 1(iii). In this case $R(z)=1 /(1-q z)$ and $z_{0}=\left(p-\sqrt{p^{2}+4(b \lambda+q)}\right) /(2(b \lambda+q))$. 


\subsection{System Characteristics}

We compute the following system characteristics: the stationary mean service cycle, the mean number of impatient customers, and the system intensity.

(i) Let $\boldsymbol{\beta}=\left(\beta_{i} ; i \geq 0\right)$ and $\boldsymbol{\rho}=\left(\rho_{i} ; i \geq 0\right)$ such that $\beta_{i}=E^{i}\left[T_{n+1}-T_{n}\right]$ and $\rho_{i}=a \lambda \beta_{i}$. Then it can be shown that

$$
\beta_{i}=E^{i}\left[T_{1}\right]= \begin{cases}\frac{1}{\lambda} \bar{\nu}^{(i)}+b, & i<r, \\ b, & i \geq r,\end{cases}
$$

and

$$
\begin{aligned}
\rho_{i} & = \begin{cases}a \bar{\nu}^{(i)}+\rho, & i<r, \\
\rho, & i \geq r,\end{cases} \\
\boldsymbol{P} \boldsymbol{\beta} & =\frac{1}{\lambda} \sum_{i<r} \bar{\nu}^{(i)} p_{i}+b, \\
\boldsymbol{P} \boldsymbol{\rho} & =a \lambda \boldsymbol{P} \boldsymbol{\beta}=a \sum_{i<r} \bar{\nu}^{(i)} p_{i}+\rho .
\end{aligned}
$$

$\boldsymbol{P} \boldsymbol{\beta}$ is called the stationary mean service cycle.

(ii) Let $d$ denote the stationary mean number of disappointed customers. Then

$$
\begin{aligned}
d & =E\left[Q_{n}-r-\Delta_{n}\left(Q_{n}-r\right)\right] \\
& =\sum_{i=r}^{N}\left(i-r-\bar{g}_{i-r}\right) p_{i} .
\end{aligned}
$$

(iii) Let $\mathcal{I}$ denote the system intensity. Then

$$
\begin{aligned}
\mathcal{I} & =\boldsymbol{P} \boldsymbol{\rho}-d \\
& =a \sum_{i<r} \bar{\nu}^{(i)} p_{i}+\sum_{i=r}^{N}\left(r-i+\bar{g}_{i-r}\right) p_{i}+\rho .
\end{aligned}
$$

Note that given the equilibrium condition $\rho<r$, the system intensity and the server capacity $r$ are equal:

$$
\mathcal{I}=r .
$$

This follows directly from (4.7), (4.12) and (4.14). 


\section{CONTINUOUS TIME PARAMETER PROCESS}

\subsection{Probability Generating Function}

The process $Q(t)$ is a semi-regenerative process with conditional regenerations at points $T_{n}, n \geq 0$. As in Dshalalow and Tadj [6], it is ergodic if and only if $\rho<r$. Let $\delta_{i}(t)=P\left\{Z(t)=i, T_{1}>t\right\}$, where $Z(t)$ is the input process. Then direct probability arguments give the elements of the semi-regenerative kernel $K(t)$

$$
\begin{aligned}
& K_{i j}(t)=\delta_{j-i}(t), \quad 0 \leq i \leq j<r, \\
& K_{i j}(t)=\sum_{s=r-i}^{j-i} \int_{0}^{t} \phi_{i}(s+i, t-u) \delta_{j-i-s}(u)[1-B(u)] \mathrm{d} u, \quad 0 \leq i<r \leq j,
\end{aligned}
$$

$K_{i j}(t)=\sum_{s=0}^{r-i} \delta_{j-r-s}(t)[1-B(t)] g_{s}^{(i-r)}, \quad r \leq i \leq j$,

$K_{i j}(t)=\delta_{j-i}(t)[1-B(t)], \quad N<i \leq j$,

$K_{i j}(t)=0, \quad 0 \leq j<i$,

where $\phi_{i}$ denotes the density of the joint probability distribution function of the random variable $S_{\nu_{0}}$ and the instant $\tau_{\nu_{0}}$ of the first excess above level $r$ by the queueing process $\{Q(t)\}$. The following is the main result of this section.

Proposition 5 Denote by $\pi=\left(\pi_{i}, i \geq 0\right)$ the steady-state vector of probabilities for the semi-regenerative process $\{Q(t)\}$ and let $\pi(z)$ be the probability generating function of the vector $\pi$. Then $\pi(z)$ is given by

$$
\begin{aligned}
\lambda \boldsymbol{P} \boldsymbol{\beta} \pi(z)= & P(z) \Delta(z)+F(z) \sum_{i=0}^{r-1} \tilde{G}_{i}(z) p_{i} \\
& +\sum_{i=r}^{N}\left[\sum_{s=0}^{i-r} g_{s}^{(i-r)} u_{s}^{(i)}(z)-z^{i} \Delta(z)\right] p_{i},
\end{aligned}
$$


where

$$
\begin{aligned}
\Delta(z) & =\frac{1-F(z)}{1-a(z)}, \\
u_{s}^{(i)}(z) & =\mathcal{D}_{x}^{i-r-s}\left\{\frac{1}{1-x}[\Delta(z)-x \Delta(x z)]\right\},
\end{aligned}
$$

and the operator $\mathcal{D}_{x}^{i-r-s}$ is defined by (3.1).

Proof By the main convergence theorem for semi-regenerative processes

$$
\pi(z)=\frac{\mathbf{P} \boldsymbol{h}(z)}{\boldsymbol{P} \boldsymbol{\beta}},
$$

where $\boldsymbol{h}(z)=\left(h_{i}(z), i \geq 0\right)$ and $h_{i}(z)$ is the generating function of the $i$ th row of the integrated semi-regenerative kernel. So having already computed the elements $K_{i j}(t)$, we find the elements $h_{i j}=\int_{0}^{\infty} K_{i j}(t) \mathrm{d} t$ of the integrated semi-regenerative kernel (by routine calculus). We then find the generating functions $h_{i}(z)$ and use relation (5.9) to get relation (5.6).

\subsection{Example}

Assume that customers arrive singly, i.e., $a(z)=z$. Then

$$
\begin{aligned}
\mathcal{D}_{x}^{i}\left\{\frac{1}{1-x}[\Delta(z)-x \Delta(x z)]\right\}=\frac{1}{1-z} & {\left[\sum_{m=0}^{i} \frac{B^{*(i-m)}(\lambda)}{(i-m) !}(-\lambda)^{i-m}\right.} \\
& \left.\times z^{i}\left(z^{-m}-1\right)+z^{i}-F(z)\right] .
\end{aligned}
$$

Also,

$$
\tilde{G}_{i}(z)= \begin{cases}\frac{z^{i}-z^{r}}{1-z}, & i<r, \\ 0, & i \geq r .\end{cases}
$$


Applying some algebraic transformations to formula (5.6) we get

$$
\begin{aligned}
\lambda(1- & z) \boldsymbol{P} \boldsymbol{\beta} \pi(z) \\
= & {[1-F(z)] P(z)+F(z) \sum_{i=0}^{N}\left[z^{i}-z^{r} g_{i}(z)\right] p_{i} } \\
& +\sum_{i=r}^{N} \sum_{s=0}^{i-r} g_{i-r-s}^{(i-r)} \sum_{p=0}^{s}\left(z^{-p}-1\right)(-\lambda)^{s-p} \frac{B^{*(s-p)}(\lambda)}{(s-p) !} p_{i} z^{i} .
\end{aligned}
$$

\subsection{System Characteristics}

We compute the following system characteristics: the mean idle period, the mean busy period, the mean output rate, the mean (actual and total) input rates, and the mean impatience rate.

(i) By probability arguments, the expected length of an idle period in equilibrium

$$
\bar{I}=\frac{\sum_{i=0}^{r-1} p_{i} \bar{\nu}^{(i)}}{\sum_{i=0}^{r-1} p_{i}} .
$$

The probability that the server idles in the stationary mode is

$$
\sum_{i=0}^{r-1} \pi_{i}=\frac{\bar{I}}{\bar{I}+\bar{B}}
$$

where $\bar{B}$ is the busy period which can be expressed therefore as

$$
\bar{B}=\frac{\sum_{i=r}^{\infty} \pi_{i}}{\sum_{i=0}^{r-1} \pi_{i}} \bar{I}
$$

(ii) The mean output rate defined $\mathcal{O}=\lim _{t \rightarrow \infty} E^{i}[S([0, t])] / t$, where $S([0, t])$ is the number of customers completely processed during the time interval $[0, t]$. It can be shown by considerations similar to ones in Dshalalow [5], to be

$$
\mathcal{O}=\frac{r}{\boldsymbol{P} \boldsymbol{\beta}} .
$$


(iii) The (actual) mean input rate $\kappa$ of any random process is (see Dshalalow [5])

$$
\kappa=\frac{\mathcal{I}}{\boldsymbol{P} \boldsymbol{\beta}} .
$$

Similarly, the (total) mean input rate can be shown to be

$$
\bar{\lambda}=\frac{\boldsymbol{P} \rho}{\boldsymbol{P} \boldsymbol{\beta}} .
$$

It follows that the mean impatience rate $\delta=\bar{\lambda}-\kappa$ is given by

$$
\delta=\frac{d}{\boldsymbol{P} \boldsymbol{\beta}} .
$$

Note that given the equilibrium condition $\rho<r$, the (actual) mean input rate $\kappa$ and the mean output rate $\mathcal{O}$ are equal, that is $\kappa=\mathcal{O}$. This follows from (4.12), (4.13) and (4.15).

In this paper we considered a queueing system where customers arrive in batches of random size and are served in batches of a fixed size. Impatience of unserved customers is taken into consideration. An ergodicity condition and steady-state probabilities are derived. Various system characteristics are also computed.

\section{References}

[1] Abolnikov, L. and Dshalalow, J.H., A first passage problem and its applications to the analysis of a class of stochastic models, J. App. Math. Stoch. Anal., 5(1), 83-98, 1991.

[2] Abolnikov, L., Dshalalow, J.H. and Dukhovny, A., On some queue length controlled stochastic processes, J. App. Math. Stoch. Anal., 3(4), 227-244, 1990.

[3] Abolnikov, L. and Dukhovny, A., Markov chains with transition delta matrix: Ergodicity conditions, invariant probability measures and applications, J. App. Math. Stoch. Anal., 4(4), 333-356, 1991.

[4] Dshalalow, J.H., Excess level processes in queueing, In: Advances in Queueing. Theory, Methods, and Open Problems, Ed., Dshalalow, J.H. Boca Raton, FL, 243262, 1995.

[5] Dshalalow, J.H., On modulated random measures, J. Appl. Math. Stoch. Anal., 4(4), 305-312, 1991.

[6] Dshalalow, J.H. and Tadj, L., On Applications of first excess level random processes to queueing systems with random server capacity and capacity dependent service time, Stochastic and Stochastic Reports, 45, 45-60, 1993. 


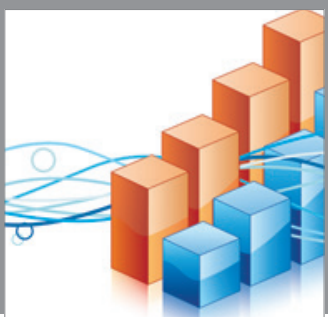

Advances in

Operations Research

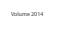

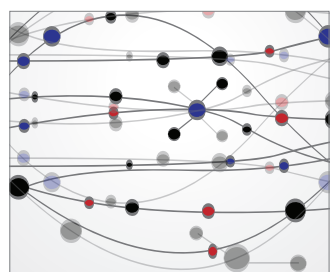

\section{The Scientific} World Journal
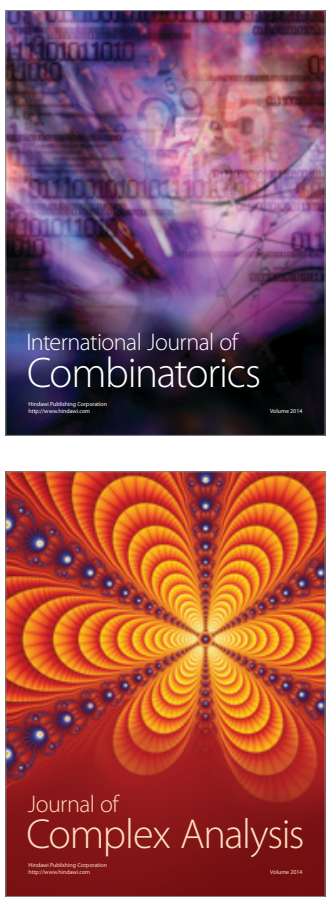

International Journal of

Mathematics and

Mathematical

Sciences
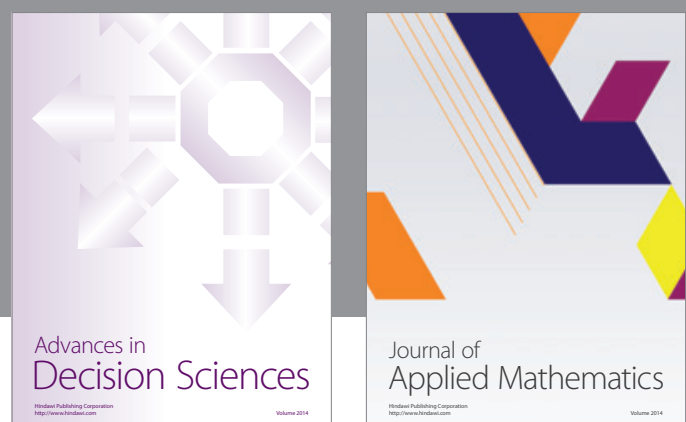

Journal of

Applied Mathematics
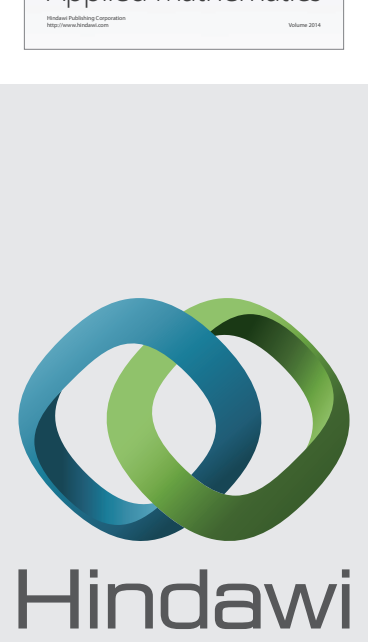

Submit your manuscripts at http://www.hindawi.com
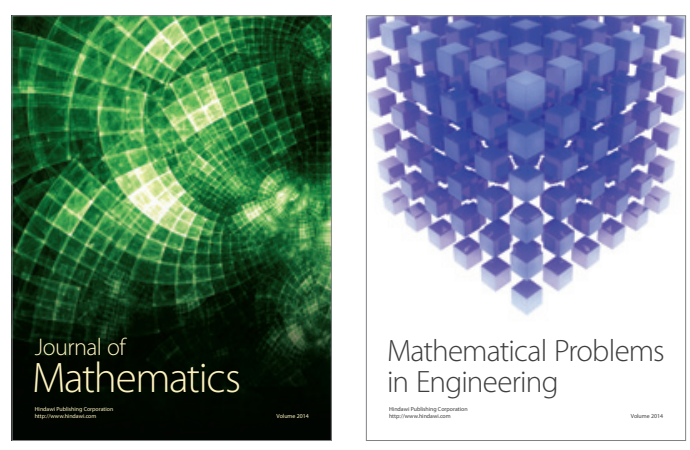

Mathematical Problems in Engineering
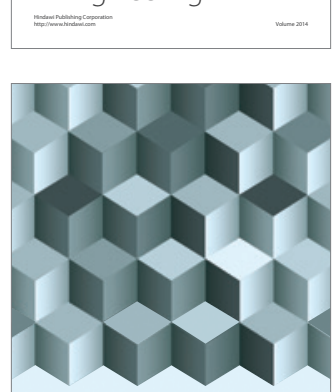

Journal of

Function Spaces
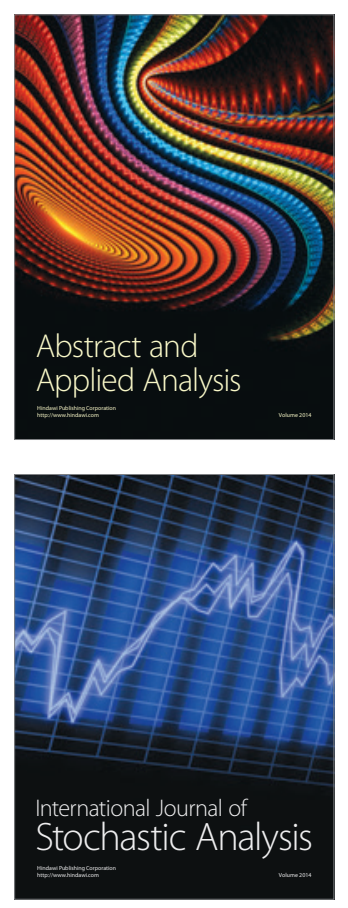

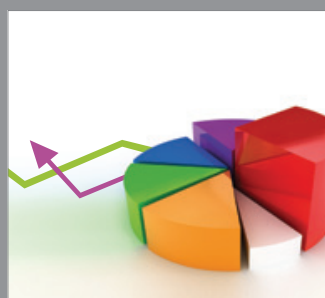

ournal of

Probability and Statistics

Promensencen
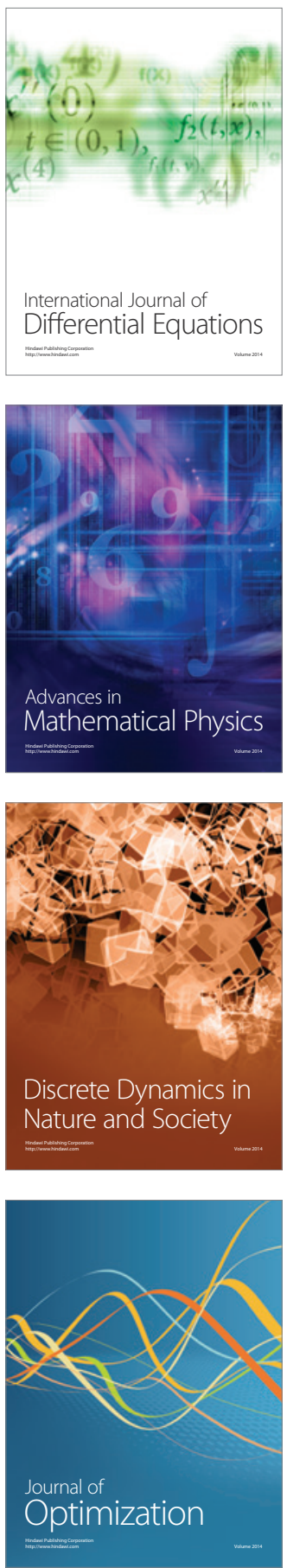